\title{
ANÁLISE DOS FATORES ASSOCIADOS AO ÓBITO FETAL INTRAUTERINO NOS CASOS ATENDIDOS EM UM HOSPITAL PÚBLICO
}

\author{
ANÁLISIS DE LOS FACTORES ASOCIADOS A LA MUERTE FETAL INTRAUTERINA EM LOS CASOS \\ TRATADOS EM UM HOSPITAL PÚBLICO
}

\section{ANALYSIS OF THE FACTORS ASSOCIATED WITH INTRAUTERINE FETAL DEATH IN A PUBLIC HOSPITAL.}

\author{
Roselane Gonçalves ${ }^{1}$, Jacqueline Isaac Machado Brigagão², Bruna Martins Cardoso Silva ${ }^{3}$, \\ Caroline Gonçalves de Oliveira ${ }^{4}$, Jenifer Daniele de Lima Santos ${ }^{5}$
}

\section{RESUMO}

O objetivo dessa pesquisa foi identificar e analisar os fatores associados aos óbitos fetais intrauterinos a partir dos registros nos prontuários médicos, de um hospital público da região metropolitana de São Paulo, no período de 2010 a 2015. Método: Realizou-se estudo exploratório descritivo e retrospectivo. Resultados: A análise de prontuários permitiu identificar que a idade gestacional, quando da ocorrência do óbito fetal, majoritariamente estava entre 28 e 37 semanas, o peso da maioria dos bebês estava acima de $2000 \mathrm{~g}$ e as patologias predominantes foram as síndromes hipertensivas. Conclusões: Dada a possibilidade de identificação precoce e monitoramento de tais patologias pela atenção básica na assistência ao pré-natal, concluiu-se que é fundamental investir nestes serviços, com vistas à prevenção do óbito fetal, bem como na melhoria dos registros nos prontuários e na implementação de ações de vigilância do óbito fetal.

DESCRITORES: Natimortos; Declaração de óbito; Epidemiologia Descritiva; Enfermagem Obstétrica; Obstetrícia.

\section{RESUMEN}

El objetivo de esta investigación fue el de identificar y analizar los factores asociados a las muertes fetales intrauterinas desde los registros médicos de un hospital público en la región metropolitana de São Paulo, de 2010 a 2015. Método: Este es un estudio exploratorio descriptivo y retrospectivo. Resultados: El análisis de los registros médicos permitió identificar que la edad gestacional al momento de la muerte fetal estuvo mayoritariamente entre 28 y 37 semanas, el peso de la mayoría de los bebés fue superior a $2000 \mathrm{~g}$ y las patologías predominantes fueron las síndromes hipertensivos. Conclusión: Dada la posibilidad de identificación temprana y monitoreo de tales patologías por la atención primaria en la atención prenatal es esencial invertir en atención prenatal, a fin de prevenir la muerte fetal, así como mejorar los registros en las fichas clínicas e implementar acciones para monitoreo de la muerte fetal.

DESCRIPTORES: Mortinatos; Certificado de Defunción; Epidemiología Descriptiva; Enfermería Obstétrica; Obstetricia.

1 Enfermeira Obstétrica. Professora Doutora da Escola de Artes, Ciências e Humanidades - Universidade de São Paulo (EACH-USP), docente do Curso de Obstetrícia da EACH-USP, São Paulo, SP, Brasil, (roselane@usp.br). ORCID: 0000-00031861-0985. Endereço: Av. Francisco Rodrigues Filho, 1902 - Casa 5 - Nova Mogilar - CEP: 08773-380 - Mogi das Cruzes - São Paulo. Telefone: 11-9-4711-6410.

2 Psicóloga Social. Professora Doutora da Escola de Artes, Ciências e Humanidades - Universidade de São Paulo (EACH -USP), docente do Curso de Obstetrícia da EACH-USP, São Paulo, SP, Brasil, (jac@usp.br). ORCID: 0000-0002-5566-8839.

3 Obstetriz. Formada pela Escola de Artes, Ciências e Humanidades - Universidade de São Paulo (EACH-USP), Especializanda em Saúde Coletiva pelo Instituto de Saúde, IS-SES/SP. São Paulo, SP, Brasil, (bruna.martins.silva@usp.br). ORCID: 0000-0002-6720-8901.

4 Obstetriz. Formada pela Escola de Artes, Ciências e Humanidades - Universidade de São Paulo (EACH-USP), São Paulo, SP, Brasil, (carolineoliveira@outlook.com). ORCID: 0000-0001-8875-2300.

5 Obstetriz. Formada pela Escola de Artes, Ciências e Humanidades - Universidade de São Paulo (EACH-USP), Especialista em Saúde Pública pelo Instituto de Saúde de São Paulo, São Paulo, SP, Brasil, (jeniferlimas@outlook.com). ORCID: 00000001-9133-7765. 


\section{ABSTRACT}

The objective of this research was to identify and analyze the factors associated with intrauterine fetal deaths from the medical records of a public hospital in the metropolitan region of São Paulo, between 2010 and 2015. Method: This is a descriptive and retrospective exploratory study. Results: The analysis of the medical records allowed us to identify that the gestational age at the time of fetal death was mostly between 28 and 37 weeks, the weight of the majority of babies was over 2000 grams and the predominant pathologies were hypertensive syndromes. Given the possibility of early identification and monitoring of such pathologies in prenatal care these deaths could have been avoided. Conclusion: It is essential to invest in prenatal care, in order to prevent fetal death, as well as to improve clinical records and implement actions to monitor fetal death.

KEYWORDS: Stillbirth; Death Certificates; Epidemiology Descriptive; Obstetric Nursing; Obstetric Nursing; Obstetrics. 


\section{INTRODUÇÃO}

O Óbito Fetal Intrauterino (OFIU) é um evento atendido em grande parte dos serviços de saúde brasileiros, mas a qualidade de informação sobre esses casos ainda é baixa ${ }^{1,2}$. Heazel et $a^{\beta}$ destacam que os custos indiretos e intangíveis da natimortalidade são substanciais e a subvalorização do ônus de uma perda fetal afeta diretamente a capacidade de enfrentamento da situação pelas plataformas políticas e programas de saúde nacionais e internacionais.

De acordo com o Ministério da Saúde ${ }^{4}$, óbito fetal é a morte de um produto da concepção, antes da sua expulsão ou da sua extração completa do corpo da mãe, com peso ao nascer igual ou superior a 500 gramas. Quando não se dispuser de informações sobre o peso ao nascer, serão considerados aqueles com idade gestacional de 22 semanas (154 dias) de gestação ou mais, ou ainda, quando nem o peso ao nascer nem a idade gestacional forem conhecidos, considerar-se-á aqueles com comprimento corpóreo de 25 centímetros cabeça-calcanhar ou mais. Quanto às terminologias utilizadas, Montenegro, Silva, Rocha e Filho ${ }^{1}$ comentam que, os termos natimortalidade e natimorto são mais utilizados na literatura em saúde.

Internacionalmente não há consenso sobre as definições quanto a idade gestacional e peso fetal para os casos de óbito fetal intrauterino (OFIU). Para o French National College of Gynecologists and Obstetricians o OFIU é definido como a cessação espontânea da atividade cardíaca fetal ocorrendo antes ou durante o parto, após 14 semanas de gestação, embora o limiar de 22 ou 28 semanas ou mais sejam comumente usados em muitos países ${ }^{5}$. Segundo o American College of Obstetricians and Gynecologists - ACOG ${ }^{6}$ se considera a idade gestacional maior ou igual a 20 semanas ou peso fetal maior ou igual a 350 gramas. De acordo com o Centers for Disease Control and Prevention/National Center for Health Statistics ${ }^{7}$, trata-se do feto que nasce sem sinais de vida, indicado pela ausência de respiração, batimentos cardíacos, pulsação do cordão umbilical ou movimentos definidos da musculatura voluntária. A Organização Mundial de Saúde estabelece como parâmetro para comparação internacional que, são considerados natimortos os bebês que nascem sem sinal de vida a partir das 28 semanas de gestação ${ }^{8}$.

A perda fetal é um problema de saúde pública dado seu impacto nos indicadores de saúde perinatal e na qualidade de vida das pessoas envolvidas, em espe- cial às mulheres e seus familiares. Assim é fundamental visibilizar o problema, propor condutas que possam auxiliar na redução de suas taxas, bem como buscar entender os fatores associados a essa problemática, tais como as condições de saúde da população de mulheres que vivenciam gestações de risco habitual ou específico e a qualidade da assistência obstétrica. Nesse sentido é fundamental para o campo da enfermagem obstétrica ampliar a produção de conhecimentos sobre essa temática. Deste modo, esta pesquisa teve como objetivo identificar e analisar os fatores associados aos OFIU atendidos num hospital público da região metropolitana de São Paulo, no período de 2010 a 2015, a partir dos registros nos prontuários médicos.

\section{MÉTODO}

Trata-se de um estudo quantitativo descritivo e retrospectivo, que analisou os prontuários médicos dos casos de natimortos registrados no Serviço de Arquivo Médico e Estatística (SAME) de um hospital público localizado em um dos municípios da região metropolitana de São Paulo, no período de 2010 a $2015^{(6 *)}$. O projeto de pesquisa foi autorizado pelo Comitê de Ética do hospital e pelo Comitê de Ética e Pesquisa da Escola de Artes, Ciências e Humanidades da Universidade de São Paulo - EACH/USP (Parecer CAAE 40382114.5.0000.5390).

A população do estudo foi composta de 14.094 prontuários de partos realizados no hospital no período supracitado sendo que, a amostra foi constituída de todos os prontuários de OFIU identificados nestes registros do SAME. Foram excluídos todos os casos de óbito fetal ocorridos nos períodos intraparto ou morte neonatal. Foram identificados e analisados 120 (cento e vinte) prontuários de mulheres com o diagnóstico de OFIU, ou seja, morte fetal no período antenatal.

Para a coleta de dados nos 120 prontuários de óbito fetal, utilizou-se um instrumento estruturado que incluiu oito variáveis de pesquisa: total anual de OFIU; idade materna; escolaridade; estado civil; informações do pré-natal; diagnósticos associados à gravidez; dados do parto atual (evolução do trabalho de parto, tipo de parto, indução/condução do trabalho de parto); informações sobre o natimorto (peso). As variáveis relativas à estatura e sexo do feto, bem como a escolaridade

6 Durante todo o ano de 2014 o hospital esteve fechado para reforma do Centro Obstétrico, e não foram realizados partos nesse ano, por isso não há dados relativos a 2014, e todas as vezes que nos referimos ao período de 2010 a 2015, entenda-se, 2010, 2011, 2012, 2013, 2015. 
materna e as informações relativas ao pré-natal foram excluídas desse trabalho porque, na maioria dos prontuários analisados elas não estavam descritas. Os dados foram coletados pelas pesquisadoras no período de outubro de 2015 a fevereiro de 2016 . Utilizou-se análise estatística descritiva simples.

\section{RESULTADOS}

O cálculo do coeficiente de natimortalidade (CNM) do hospital foi realizado utilizando somente os casos de óbito fetal que ocorreram no período antenatal. O coeficiente foi de 5,15:1000 em 2010; 8,35:1000 em 2011; 11,5:1000 em 2012; 6,1:1000 em 2013 e 11,9:1000 em $2015^{(*)}$.

No que se refere às mulheres admitidas com o diagnóstico de OFIU, a tabela 1 apresenta os dados sociais e obstétricos, sendo as variáveis pesquisadas: idade materna; estado civil; número de gestações; paridade e idade gestacional. Na maioria dos prontuários analisa-

\begin{tabular}{ccc}
$\begin{array}{c}\text { Dados obsté tricos e sociais da } \\
\text { mulher }\end{array}$ & N & $\%$ \\
\hline Faixa etária materna (anos) & 17 & 14.2 \\
0 à 18 & 43 & 35.8 \\
19 à 25 & 40 & 33.3 \\
26 à 35 & 20 & 16.7 \\
$>36$ anos & & \\
Estado civil & 51 & 42,5 \\
$\quad$ Casada & 29 & 24,2 \\
$\quad$ Solteira & 20 & 16,7 \\
União estável & 1 & 0,8 \\
Divorciadas & 19 & 15,8 \\
Não informado & & \\
Paridade $\quad$ Nulípara & 47 & 39.1 \\
1 & 26 & 21.6 \\
2 & 16 & 13.3 \\
$\geq 3$ & 22 & 18,2 \\
Não informado & 9 & 7.5
\end{tabular}

\begin{tabular}{|c|c|c|}
\hline \multicolumn{3}{|c|}{ pelas au- } \\
\hline 22 à 27 semanas efodias & S do SAMF & 25.8 \\
\hline 28 à 36 semanas e 6 dias & UO & 36.7 \\
\hline$>37$ semanas & 41 & 34.2 \\
\hline Não informado & 4 & 3.3 \\
\hline
\end{tabular}

A tabela 1 indica que os casos de óbito fetal ocorreram em todas as faixas etárias do ciclo reprodutivo feminino. Na maioria dos casos, as mulheres tinham idade entre 19 a 35 anos (69,1\%). Quanto ao estado civil, mais da metade $(59,2 \%)$ eram casadas ou viviam em união estável, em 19 prontuários (15.8\%) não havia registro desta informação. Quanto à paridade, a maioria era nulípara $(39,1 \%)$ seguida pelas mulheres que estavam vivendo o segundo parto (21.6\%). Em 7,5\% dos prontuários não havia registro sobre a paridade.
Tabela 2 - Distribuição das patologias maternas na população de óbitos fetais conforme a idade gestacional (Razão e Proporção) - São Paulo - 2010 a 2015.

\begin{tabular}{|c|c|c|c|c|c|c|}
\hline \multirow{2}{*}{$\begin{array}{l}\text { Patologias } \\
\text { matemas }\end{array}$} & \multicolumn{4}{|c|}{ Idade Ge stacional } & \multicolumn{2}{|c|}{ Total } \\
\hline & $\begin{array}{c}22 \text { à } 27 \mathrm{~s} \\
\text { e } 6 \mathrm{~d}\end{array}$ & $\begin{array}{c}28 \text { à } 36 s \\
\text { e } 6 d\end{array}$ & $\geq 37 d$ & $\mathrm{~N} / \mathrm{l}$ & $\mathrm{N}$ & $\%$ \\
\hline $\begin{array}{l}\text { Síndromes } \\
\text { Hipertensivas }\end{array}$ & 10 & $15^{\star}$ & $14^{*}$ & - & 39 & 28.3 \\
\hline $\begin{array}{l}\text { Diabetes } \\
\text { Mellitus }\end{array}$ & $2^{*}$ & 2 & $3^{*}$ & - & 7 & 5.1 \\
\hline ITU & 1 & 2 & 3 & - & 6 & 4.3 \\
\hline Tabagismo & $2^{*}$ & - & $3^{*}$ & - & 5 & 3.6 \\
\hline Anemia & 1 & 1 & 1 & - & 3 & 2.2 \\
\hline Obesidade & - & - & $2^{*}$ & - & 2 & 1.4 \\
\hline DPP & - & $1^{*}$ & - & - & 1 & 0.7 \\
\hline Drogas ilicitas & - & - & 1 & - & 1 & 0.7 \\
\hline Placenta previa & 1 & - & - & - & 1 & 0.7 \\
\hline Sífilis & - & 1 & - & - & 1 & 0.7 \\
\hline Toxoplasmose & 1 & - & - & - & 1 & 0.7 \\
\hline Hipertireoidismo & - & - & 1 & - & 1 & 0.7 \\
\hline Cardiopatia & 1 & - & - & - & 1 & 0.7 \\
\hline Mioma & - & 1 & - & - & 1 & 0.7 \\
\hline Epilepsia & - & 1 & - & - & 1 & 0.7 \\
\hline Nenhum agravo & 16 & 25 & 22 & 4 & 67 & 48.5 \\
\hline Total & 35 & 49 & 50 & 4 & $138^{*}$ & - \\
\hline
\end{tabular}

* Indica os casos em que havia mais de uma patologia materna para um mesmo caso de óbito

N/l: não informado.

Fonte: Adaptado pelas autoras. Dados do SAME.

Os óbitos fetais podem estar associados às patologias maternas durante a gestação. $\mathrm{Na}$ análise dos prontuários buscou-se identificar as principais patologias maternas registradas e a idade gestacional em que ocorreu o OFIU. As síndromes hipertensivas específicas ou não da gravidez (pré-eclâmpsia, hipertensão arterial crônica e hipertensão arterial adquirida na gestação) foram predominantes $(28,3 \%)$, principalmente nas idades gestacionais entre 28 e 36 semanas e 6 dias, seguidas da diabetes mellitus (5,1\%); a placenta prévia, a sífilis, a toxoplasmose, a cardiopatia e o mioma foram listados na tabela porque apareceram em pelo menos um dos prontuários analisados. O único caso de DPP ocorreu em associação com a síndrome hipertensiva/ pré-eclâmpsia quando a idade gestacional estava entre 28 a 36 semanas e 6 dias. Em outros dois casos de síndrome hipertensiva/ pré-eclâmpsia encontrou-se associação com a obesidade, ambos identificados após 37 semanas gestacionais, além da anemia ( 1 caso com idade gestacional entre 22 a 27 semanas e 6 dias), diabetes ( 2 casos identificados nas seguintes idades ges- 
tacionais: 22 a 27 semanas e 6 dias, > 37 semanas) e infecção do trato urinário (1 caso, idade gestacional entre 28 a 36 semanas e 6 dias). O tabagismo apareceu associado à outras condições, tais como a pré-eclâmpsia (dois casos, ambos na idade gestacional entre 22 e 27 semanas e seis dias) e a infecção do trato urinário, 1 caso diagnosticado no mesmo período gestacional. Houve dois casos em que três patologias estiveram associadas: ITU, pré-eclâmpsia e mioma (1 caso com idade gestacional entre 28 a 36 semanas e 6 dias) e tabagismo e pré-eclâmpsia e diabetes (1 caso com idade gestacional $>37$ semanas).

Para ampliar o entendimento sobre as possíveis causas dos óbitos fetais foram levantados os registros feitos pelo médico na cópia da Declaração de Óbito (DO) que estava arquivada no prontuário (tabela 3).

Tabela 3 - Distribuição das causas atribuídas ao OFIU segundo registros na declaração de óbito fornecida pelo hospital (Razão e Proporção) - São Paulo - 2010 a 2015.

\begin{tabular}{ccc}
\hline Causa OFIU & \multicolumn{2}{c}{ Total } \\
\hline Anóxia Intrauterina & 90 & 58.8 \\
Insuficiência & 22 & 14.4 \\
uteroplacentária & 16 & 10.4 \\
DPP & 15 & 9.8 \\
Pré-eclampsia & 4 & 2.6 \\
Hipóxia intrauterina & 4 & 2.6 \\
Sofrimento fetal agudo & 2 & 1.3 \\
Oligoâmnio severo & 2 & 100 \\
Total* & 153 &
\end{tabular}

* Houve mais de um diagnóstico num mesmo de caso de óbito fetal

Fonte: Adaptado pelas autoras. Dados do SAME.

Na tabela 3 é possível observar a distribuição das causas de OFIU com destaque para a anóxia intrauterina, o diagnóstico mais utilizado nas declarações de óbito.

Afim de observar se o crescimento dos fetos estava condizente com a idade gestacional buscou-se relacionar o peso ao nascer e a idade gestacional conforme demonstra a tabela 4 .
Tabela 4 - Distribuição dos casos de acordo com as variáveis relacionadas à idade gestacional e o peso do natimorto (Razão e Proporção) - São Paulo - 2010 a 2015.

\begin{tabular}{|c|c|c|c|c|c|c|}
\hline \multirow{2}{*}{$\begin{array}{l}\text { Peso do } \\
\text { Natimorto }\end{array}$} & \multicolumn{4}{|c|}{ Idade gestacional em semanas } & \multicolumn{2}{|c|}{ Total } \\
\hline & 22 à $27 \mathrm{~s} \mathrm{e} 6 \mathrm{~d}$ & 28 à $36 \mathrm{~s}$ e $6 \mathrm{~d}$ & $\begin{array}{c}\geq 37 \\
\text { semanas }\end{array}$ & $\mathrm{N} / \mathrm{I}$ & $\mathrm{N}$ & $\%$ \\
\hline 500 à $1000 \mathrm{~g}$ & 25 & 7 & - & 2 & 34 & 28.3 \\
\hline 1001 à $2000 \mathrm{~g}$ & 4 & 25 & 3 & - & 32 & 26.6 \\
\hline 2001 à $3000 \mathrm{~g}$ & - & 7 & 20 & 1 & 28 & 23.3 \\
\hline 3001 à $4000 \mathrm{~g}$ & - & 5 & 17 & 1 & 23 & 19.1 \\
\hline$\geq 4001 \mathrm{~g}$ & - & - & 1 & - & 1 & 0.8 \\
\hline Não informado & 2 & - & - & - & 2 & 1.6 \\
\hline Total & 31 & 44 & 41 & 4 & 120 & 100 \\
\hline
\end{tabular}

$\mathrm{N} / \mathrm{I}$ : não informado.

Fonte: Adaptado pelas autoras. Dados do SAME.

Os registros indicam que em $43,3 \%$ dos casos de OFIU, os bebês tinham peso superior a $2000 \mathrm{~g}$ e chegaram ao terceiro trimestre gestacional ( $28^{a}$ em diante).

Quanto aos tipos de parto e as condutas obstétricas nos casos de OFIU buscou-se relacionar os tipos de parto com a paridade das mulheres, como demonstra a tabela 5 .

Tabela 5 - Distribuição dos casos de acordo com as variáveis relacionadas a paridade e tipo de parto (Razão e Proporção) São Paulo - 2010 a 2015.

\begin{tabular}{|c|c|c|c|c|c|c|}
\hline \multirow[b]{2}{*}{ Paridade } & \multicolumn{4}{|c|}{ Tipo de Parto } & \multicolumn{2}{|c|}{ Total } \\
\hline & Normal & Cesariana & Fórceps & $N / 1$ & $\mathbf{N}$ & $\%$ \\
\hline Nulípara & 31 & 15 & 1 & - & 47 & 39.2 \\
\hline 1 & 19 & 7 & - & - & 26 & 21.7 \\
\hline 2 & 9 & 7 & - & - & 16 & 13.3 \\
\hline$\geq 3$ & 5 & 16 & - & 1 & 22 & 18.3 \\
\hline Não informado & 8 & 1 & - & - & 9 & 7.5 \\
\hline Total & 72 & 46 & 1 & 1 & 120 & 100 \\
\hline
\end{tabular}

N/I: Não informado

Fonte: Adaptado pelas autoras. Dados do SAME.

Conforme a tabela 5 indica o parto vaginal foi predominante e as cesarianas foram realizadas em $38,3 \%$ dos casos e em $0,8 \%$ o parto foi instrumental. 


\section{DISCUSSÃO}

O CNM está abaixo do coeficiente brasileiro que, segundo Barbeiro et al ${ }^{9}$, foi 10,7:1000 no mesmo período desse estudo, porém, ainda está acima dos CNM dos países desenvolvidos. Segundo estes autores, nos Estados Unidos, a taxa de morte fetal em 2012 foi de 6,05:1000 nascimentos e tem se mantido estável ao longo do tempo. De acordo com Montenegro, Silva, Rocha e Filho ${ }^{1}$, nos países desenvolvidos, a taxa de natimortalidade estimada é de 4,2 e 6,8 por 1000 nascimentos enquanto nos países em desenvolvimento é de 20 a 32 por 1000 nascimentos. Opsiøn; Vogto ${ }^{10}$, corroboram esses resultados e argumentam que as taxas estimadas de morte fetal pela Organização Mundial da Saúde (OMS) variam no mundo e indicam que o coeficiente é 6,1 por 1000 na região europeia e 28,1 por 1000 nas regiões do continente africano. Os números encontrados no hospital estudado também estavam acima dos coeficientes dos países desenvolvidos nos anos de 2011, 2012 e 2015. Vale ressaltar que as diferentes taxas entre os países devem ser analisadas partindo da perspectiva de que a caracterização do OFIU pode variar a depender da classificação utilizada por cada país.

Sobre dados obstétricos, a idade gestacional foi classificada de acordo com os parâmetros estabelecidos pela OMS e do CID -10: $25.8 \%$ dos OFIU eram extremamente prematuros - entre 22 a 27 semanas e seis dias-; $36.7 \%$ prematuridade intermediária - entre 28 a 36 semanas e 6 dias de gestação- e 34.2\% a termo - acima de 37 semanas $^{11}$.

A faixa etária de maior ocorrência de óbitos fetais coincide com a faixa etária em que ocorre a maioria dos partos no Estado de São Paulo ${ }^{12}$. Os achados deste estudo coincidem com o estudo francês onde se encontrou a predominância de casos de OFIU na faixa etária materna entre 19 a 42 anos $^{5}$.

Quanto as complicações obstétricas relacionadas ao óbito fetal, apesar de Bukowski et a/ ${ }^{13}$ terem encontrado relação entre as complicações maternas e feto-anexiais, em $24 \%$ dos casos os OFIU foram considerados de causas idiopáticas.

No estudo de Montenegro, Silva, Rocha e Filho ${ }^{1}$ as doenças maternas representam cerca de $10 \%$ da natimortalidade nas gestações a termo, sendo hipertensão $4 \%$ e diabetes melito $3 \%$ hipertireoidismo, doença renal, doença hemolítica perinatal, lupus eritematoso sistêmico, colestase gravídica, infecção e tabagismo. Eles concluem que de $10-40 \%$ da natimortalidade a termo permanece inexplicada, apesar de rigorosa avaliação do feto, da placenta e da mãe.

Belhomme et $a l^{5}$, em estudo envolvendo 53 casos de OFIU, encontraram que as principais causas incluíram desordens placentária $(56,6 \%)$ e vasculares $(50,9 \%)$, seguidas de $20,8 \%$ de causas desconhecidas; $11,3 \%$ doença materna; 9,4\% síndrome fosfolipídica; 7,5\% anormalidades do cordão; 5,7\% desordens inflamatórias; $1,9 \%$ pré-eclâmpsia; 1,9\% infecção; 1,9\% anormalidade fetal; 1,9\% complicação intraparto. Nenhuma causa genética foi apontada. A despeito das escassas evidências sobre as causas dos OFIU algumas condições físicas como a obesidade, a hipertensão e a diabetes parecem aumentar sua incidência ${ }^{14}$.

Especificamente sobre a hipertensão arterial na gestação os resultados dessa pesquisa estão coerentes com as pesquisas nacionais. Em revisão sistemática da literatura sobre óbitos fetais no Brasil, os distúrbios hipertensivos aparecem frequentemente como um dos fatores mais prevalentes, seja isolado ou associado a outras patologias como em alguns dos casos descritos nessa pesquisa e estão diretamente relacionados com a qualidade da assistência prestada no pré-natal ${ }^{9}$. Ainda, a ocorrência da hipertensão na gravidez relaciona-se a situações que podem levar ao crescimento intrauterino restrito por insuficiência uteroplacentária, podendo culminar com a anóxia intrauterina, além da possibilidade de descolamento prematuro de placenta.

Embora pouco incidentes nos registros dos prontuários, identificou-se dois casos de doenças infectocontagiosas: sífilis e toxoplasmose, o que indica que há falhas no pré-natal, uma vez que o rastreamento dessas doenças está previsto nas diretrizes nacionais do Ministério da Saúde para o pré-natal ${ }^{15}$.

A análise da idade gestacional em que ocorrem os OFIUs é fundamental para compreender o impacto social e epidemiológico das perdas fetais considerando que quanto mais avançada a gestação maior chance os bebês teriam de sobrevida ${ }^{16}$. Assim, quando se considera o potencial de sobrevida de um feto a partir de 28 semanas de gestação até o termo, a porcentagem de perdas assume grande vulto pois, refere-se a 85 casos $(70,9 \%)$. A maioria dos casos ocorreu no terceiro trimestre gestacional, assim como o descrito por outros autores ${ }^{5,13}$, os quais identificaram cerca de $50 \%$ dos óbitos fetais neste período, momento em que, biologicamente, os fetos estão mais maduros e com maior potencial de sobrevivência.

A apreciação dos fatores associados às perdas 
fetais torna-se obrigatória e imprescindível, tanto quanto a avaliação da qualidade do acompanhamento pré-natal ${ }^{16}$. Aspectos psicossociais devem ser considerados pois, trata-se de um período em que, para a maioria das famílias, há um grande investimento afetivo no processo de espera dos bebês, há muitas expectativas em torno do nascimento e para as mulheres e seus pares se deparar com a morte fetal nessa fase implica em lidar com uma perda muito significativa. Bertolani e Oliveira ${ }^{17}$ em seu estudo sobre as mulheres em situação de abortamento concluem que as perdas fetais trazem consigo sofrimento psíquico que persistem muitos meses após o evento da perda.

Nesse estudo analisamos também a cópia da declaração de óbito que consta dos prontuários e identificamos que a anóxia intrauterina representa a condição mórbida prevalente nas declarações. Provavelmente, por ser a que melhor pode explicar a perda fetal. Todas as demais causas listadas na tabela 3 se inter-relacionam e representam condições que levam ao estado de falta de oxigenação fetal, porém são muito inespecíficas. Todos os natimortos receberam declaração/atestado de óbito fornecido pelo médico responsável pelo atendimento obstétrico, provavelmente porque no município não há serviço de verificação de óbito (SVO). Além disso, nos prontuários analisados não foram encontradas descrições a respeito das condições do feto, não foram realizadas análises de tecidos ou anexos fetais e nem registro do encaminhamento dos natimortos para necrópsia/autópsia.

De acordo com o ACOG ${ }^{18}$ a inspeção do feto pelos profissionais e por geneticista experiente, a necrópsia e cariotipagem, o exame da placenta, a história clínica e os exames complementares da mãe são indispensáveis para o diagnóstico da causa da natimortalidade. Montenegro, Silva, Rocha e Filho ${ }^{1}$ afirmam que a investigação da natimortalidade, particularmente a necrópsia, é uma conduta a ser apresentada e discutida com a família, dada a sua importância para o diagnóstico. Se a família não possibilitar a sua realização outros procedimentos menos invasivos são indicados como a documentação fotográfica e as amostras de tecido (sangue ou pele). Afirmam ainda que, a identificação sindrômica pode ser importante para avaliação do risco em gestações subsequentes. Além dos exames específicos, conduta apropriada também deve ser seguida, no que se refere a obtenção da história obstétrica e familiar, complementada com diversos estudos laboratoriais como a citologia do líquido amniótico, de tecido placentário, de segmento do cordão umbilical, de tecido interno fetal (junção costocondral ou patela).

A detecção da causa que levou à morte fetal é muito importante, não apenas para orientar a família, como também para os registros epidemiológicos que embasam programas de saúde perinatal. Sobre isso, pesquisadores italianos, visando a criação de um procedimento universal padronizado para óbitos fetais inexplicados, desenvolveram na primeira década dos anos 2000 um protocolo de investigação dos centros cerebrais de fetos não macerados que se mostrou muito útil em revelar a fisiopatologia da morte fetal quando a autópsia padrão não é esclarecedora. No entanto, o alto custo desta metodologia tem impedido sua implementação ${ }^{19}$. Desta forma, sugere-se que sejam implementados protocolos de investigação do óbito fetal no hospital pesquisado afim de investigar as suas possíveis causas. A vigilância do óbito fetal é uma das fontes de informação para que se desenvolva políticas públicas direcionadas ao cuidado em saúde das mulheres e crianças ${ }^{20,21}$.

A tabela 4 indica que a relação entre idade gestacional e peso está dentro dos parâmetros para os bebês brasileiros ${ }^{22}$. Apenas em três casos o peso do bebê ao nascer era inferior ao esperado para a idade gestacional. Nesses três, apesar da idade gestacional ser igual ou maior a 37 semanas, os bebês pesaram entre 1001 e $2000 \mathrm{~g}$. As características de idade gestacional e peso apontam que esses bebês teriam grande probabilidade de sobrevivência não fossem as condições adversas determinantes de sua morte.

Em relação ao tipo de parto, nesse estudo, o parto vaginal foi predominante $(60 \%)$ o que está de acordo com a literatura científica, já que a recomendação para os casos de OFIU é de parto vaginal ${ }^{23}$. Nos casos em que as mulheres não entram em trabalho de parto espontâneo, não havendo contraindicações maternas, é indicado a indução do mesmo com vistas a um parto vaginal. Entre 80 a $90 \%$ das mulheres entram espontaneamente em trabalho de parto na primeira e segunda semana após o diagnóstico do OFIU. São raros os casos em que podem ocorrer distúrbios de coagulação quando o período de latência ultrapassa 4 semanas $^{1,24}$. Segundo o ACOG cesarianas não devem ser a via de parto escolhida para os casos de OFIU uma vez que este tipo de parto está associado a morbidade materna ${ }^{6}$. As evidências cientificas indicam que mesmo em mulheres que já tenham histórico de cicatriz uterina anterior o parto normal representa a melhor escolha. A indução do parto deve seguir protocolos obstétricos habituais ${ }^{1,24}$. 
Porém nesse estudo, aproximadamente $38 \%$ dos partos foram cesarianas representando um índice alto quando comparado ao estudo de Boyle et al ${ }^{25}$ que, dentre 611 casos de OFIU, apenas $93(15,2 \%)$ mulheres foram submetidas à cesariana, sendo que $43,0 \%(46)$ delas tinham histórico deste tipo de parto anterior. Além disso, no presente estudo, $32,6 \%$ das mulheres submetidas a cesariana eram nulíparas e não foram encontradas justificativas para a indicação do parto cirúrgico no prontuário. Vale ressaltar que a realização de cesarianas sem indicações tem maior potencial para trazer diversos agravos a saúde da mulher. Nos casos de OFIU esses agravos incluem, ainda, ter de lidar com os desdobramentos do período pós-cirúrgico durante o processo de elaboração do luto, retardar as possibilidades de uma nova gestação e ampliar as chances de outro parto cirúrgico na próxima gravidez.

A pesquisa possibilitou constatar que nesse hospital, apesar de haver espaços no prontuário para o registro completo dos dados socioeconômicos e do pré-natal e do parto/recém-nascido esses não são preenchidos pelos profissionais de saúde o que prejudica o acompanhamento e monitoramento desses casos, bem como alimentar outros sistemas. Estudo que analisou fichas de investigação de vigilância do óbito infantil (VOI) evidenciou que o registro completo de informações auxilia a identificação das mortes evitáveis sendo um potente meio para o monitoramento do óbito infantil ${ }^{26}$.

\section{CONCLUSÕES}

A análise dos prontuários possibilitou identificar que é fundamental investir na atenção pré-natal e na articulação entre os hospitais e os serviços da atenção básica para ampliar as ações de acompanhamento e monitoramento das gestações, em especial no terceiro trimestre gestacional, isto porque as patologias encontradas são passíveis de identificação precoce e o acompanhamento eficaz pode prevenir os óbitos fetais. Ademais é preciso investir em ações de vigilância para obter informações qualificadas sobre as possíveis causas dos óbitos fetais intrauterinos, como as análises realizadas pelo Serviço de Verificação de Óbito e o acesso a outros testes que podem vir a ampliar a investigação das causas desses óbitos. Essas informações são essenciais para a elaboração de políticas e protocolos que permitam diminuir o número de casos, bem como evitar a perda de fetos viáveis.

Além disso, ficou evidente a necessidade de investimento na qualificação dos profissionais de saúde para o registro adequado dos dados nos prontuários médicos de modo que esses possam ser utilizados em estudos retrospectivos e de acompanhamento dos casos de OFIU ao longo do tempo. 


\section{REFERÊNCIAS}

1. Montenegro CAB, Silva GCR, Rocha FL, Filho JR. Morte fetal. In: Montenegro CAB, Filho JR. Rezende Obstetricia, $13^{\mathrm{a}}$ ed. Rio de Janeiro: Guanabara Koogan; 2017. p.441-446.

2. Lansky S, França E, Leal MC. Mortalidade perinatal e evitabilidade: revisão da literatura. Rev. Saúde Pública [Internet]. 2002; [acesso em 26 abril 2018] 36(6):759-772. Disponível em: http://www.scielo.br/scielo.php? script=sci_arttext \& pid=S0034$-89102002000700017 \&$ Ing=en. DOI: http://dx.doi. org/10.1590/S0034-89102002000700017

3. Heazell AEP, Siassakos D, Blencowe H, Burden C, Bhutta ZA, Cacciatore J, et al. Stillbirths: economic and psychosocial consequences. The Lancet. 2016;387(10018):604-16. DOI: 387. 10.1016/ s0140-6736(15)00836-3

4. Ministério da Saúde (BR). Secretaria de Vigilância em Saúde. Secretaria de Atenção à Saúde (BR). Manual de vigilância do óbito infantil e fetal e do Comitê de Prevenção do Óbito Infantil e Fetal. $2^{2}$ ed. [Internet] Brasília: MS, 2009. [Acesso em 10 set 2017] Disponível em: bvsms.saude.gov.br/ bvs/publicacoes/manual_obito_infantil_fetal_2ed. pdf

5. Belhomme N, Carlan MLND, Lescoat A, LeGallou $T$, Rouget $F$, Loget $P$, et al. Investigating in utero fetal death: outcome of the internal medicine consultation. International Journal of Rheumatic Diseases. 2017;21(2)381-386. DOI:10.1056/NEJMoa040405

6. American College of Obstetricians. Management of stillbirth. ACOG Practice Bulletin n. 102. Obstet. Gynecol. 2009;113:748-61. DOI: 10.1097/ AOG.0b013e31819e9ee2

7. Centers for Disease Control and Prevention /National Center for Health Statistics. State definitions and reporting requirements for live births, fetal deaths, and induced terminations of pregnancy. Hyattsville: Maryland. Revision; 1997
8. World Health Organization. Neonatal and perinatal mortality: Country, Regional and Global estimates. Geneva, Switzerland: Library Catalouguing. WHO; 2006

9. Barbeiro FMS, Fonseca SC, Tauffer MG, Ferreira MSS, Silva FP, Ventura PM, et al. Óbitos fetais no Brasil: revisão sistemática. São Paulo: Rev. Saúde Pública. 2015;49: 22 [acesso em: 17 jul 2017] Disponivel em: http://www.scielo. br/scielo.php?script=sci_arttext\&pid=S0034$-89102015000100402 \&$ Ing $=e n \& n r m=i s o$

10. Opsion BE, Vogto C. Explaining Fetal Death - What Are the Contributions of Fetal Autopsy and Placenta Examination? Society for Pediatric Pathology: Pediatric and Developmental Pathology. 2016;19:24-30. DOI: 10.2350/15-031614-OA

11. Organização Mundial da Saúde. Constituição da Organização Mundial da Saúde: Resoluções WHA20.19 e WHA43.24 [Internet]. [acesso em: 19 jan 2018] Disponível em: http://www.datasus. gov.br/cid10/V2008/WebHelp/definicoes.html

12. Instituto Brasileiro de Geografia e Estatística. Indicadores Sociodemográficos e de Saúde no Brasil. [Internet] Rio de Janeiro: Estudos e Pesquisas - Informação Demográfica e Socioeconômica. 2009;25: 13 [acesso em: 01 set 2017] Disponível em: http://www.ibge.gov.br/english/estatistica/ populacao/indic_sociosaude/2009/indicsaude.pdf.

13. Bukowski R, Carpenter M, Conway D, Coustan D, Dudley DJ, Goldenberg RL, et al. Causes of death among stillbirths. Stillbirth Collaborative Research Network Writing Group: JAMA. 2011;306(22):245968. DOI: 10.1001/jama.2011.1823

14. Song YH, Lee GM, Yoon JM, Cheon EJ, Lee $\mathrm{SK}$, Chung SH, et al. Trends in Fetal and Perinatal Mortality in Korea (2009-2014): Comparison with Japan and the United States. [Internet] J Korean Med Sci. 2017;32:1319-1326 [acesso em: 16 agosto 2017] Disponível em: https://doi. org/10.3346/jkms.2017.32.8.1319 
15. Ministério da Saúde (BR). Secretaria de Atenção à Saúde: Departamento de Atenção Básica. Atenção ao Pré-natal de Baixo Risco. Série A. Normas e Manuais Técnicos: Cadernos de Atenção Básica, $\mathrm{n}^{\circ}$ 32. Brasília - DF. 2012.

16. Fonseca SC, Coutinho ESF. Características biológicas e evitabilidade de óbitos perinatais em uma localidade na cidade do Rio de Janeiro, 1999 a 2003. Recife: Rev. Bras. Saude Mater. Infant. 2008;8(2):171-178.

17. DOI: http://dx.doi.org/10.1590/S151938292008000200004

18. Bertolani GBM, Oliveira EM de. Mulheres em situação de abortamento: estudo de caso. São Paulo: Saude Soc. 2010;19(2):286-301. DOI: $10.1590 /$ S0104-12902010000200006

19. American College of Obstetrics Gynecologists. Evaluation of stilbirths and neonatal deaths. ACOG Committee Opnion. Obstet Gynecol. 2007;383(11):963. DOI:10.1097/01. AOG.0000263934.51252.e0

20. Roncati L, Piscioli F, Pusiol T, Lavezzi AM. Neuropathological protocol for the study of unexplained stillbirth. Folia Neuropathol. 2017;55(2):79-85. DOI: http://doi.org.ololo.sci-hub.cc/10.5114/ fn.2017.68576

21. Vanderlei LCM, Frias PG de. A vigilância do ó bito como instrumento para reduzir a invisibili dade da exclusão social e assistencial de mu Iheres e crianças. Rev. Bras. Saude Mater. Infant. [Internet]. 2017;17(4):633-634. [acesso em 26 abril 2018]. Disponível em: http:// www.scielo.br/scielo.php? script=sci arttext \&pid=S1519-38292017000400633\&lng=pt. DOI: $\quad$ http://dx.doi.org/10.1590/180693042017000400001.
22. Gaíva MAM, Lopes FSP, Ferreira SMB, Mufato LF. Óbitos neonatais de recém-nascidos de baixo peso ao nascer. Rev. Eletr. Enf. 2018;20:v20a18 [acesso em 29 out 2018]. Disponível em: https:// doi.org/10.5216/ree.v20.47222.

23. Pedreira CE, Pinto FA, Pereira SP, Costa ES. Padrões de peso ao nascimento por idade gestacional no Brasil. Rio de Janeiro: An Acad Bras Cienc. 2011;2:83. DOI: http:// dx.doi.org/10.1590/S0001-37652011005000008

24. Souza ASR, Sampaio AG. Indicação de cesarianas em óbito fetal. Rev Bras Ginecol Obstet. 2010;32(4):169-75.

25. Chakhtoura NA, Reddy UM. Management of stillbirth delivery. Seminars in Perinatology. 2015;39(6):501-504. DOI: http://dx.doi. org/10.1053/j.semperi.2015.07.016

26. Boyle A, Preslar JP, Hogue CJ, Silver RM, Reddy UM, Goldenberg RL, et al. Route of Delivery in Women With Stillbirth: Results From the Stillbirth Collaborative Research Network. Obstetrics \& Gynecology. 2017;129(4):693-698. DOI: 10.1097/ AOG.0000000000001935

27. Guimarães ALS, Oliveira CM, Bonfim CV. Completude das variáveis da ficha de investigação da vigilância do óbito infantil: análise preliminar. Revista Saúde- UNG/Ser. v. 10, n.1 (ESP), 2016. [acesso em 18 nov 2019]. Disponível em: http://revistas. ung.br/index.php/saude/article/view/2671/2065

Fonte de financiamento da pesquisa: Programa Unificado de Bolsas (PUB)- USP 